\title{
DUSP4 alleviates LPS-induced chondrocyte injury in knee osteoarthritis via the MAPK signaling pathway
}

\author{
ZHENGNAN $\mathrm{LI}^{1}$ and BOJIE CHEN ${ }^{2}$ \\ Departments of ${ }^{1}$ Sports Medicine and ${ }^{2}$ Joint Surgery, Ganzhou People's Hospital, \\ Zhanggong, Jiangxi 341000, P.R. China
}

Received April 12, 2021; Accepted July 13, 2021

DOI: $10.3892 /$ etm.2021.10837

\begin{abstract}
Knee osteoarthritis (KOA) is characterized by cartilage damage, and the associated pathogenesis is complex. The expression of dual specificity protein phosphatase 4 (DUSP4) is significantly decreased in osteoarthritis (OA); however, the specific role and mechanism underlying DUSP4 in OA are yet to be elucidated. ATDC5 cells were treated with lipopolysaccharide (LPS) to establish the cell injury model. The expression levels of DUSP4 were decreased in OA chondrocytes, demonstrated by reverse transcription-quantitative PCR and western blot analysis. Following overexpression of DUSP4 by cell transfection, Cell Counting Kit-8, ELISA, TUNEL and western blotting assays were used to detect the cell viability, oxidative stress, inflammation and apoptosis levels of LPS-induced ATDC5 cells. Overexpression of DUSP4 inhibited the activation of the MAPK signaling pathway, thereby reducing oxidative stress levels, inflammatory response and apoptosis in the OA cell model. The mechanisms underlying DUSP4 in OA were further explored following the addition of MAPK signaling pathway agonist, phorbol 12-myristate 13-acetate (PMA). The addition of PMA reversed the inhibitory effects of DUSP4 overexpression on oxidative stress, inflammatory response and apoptosis in cells. In summary, DUSP4 alleviated LPS-induced chondrocyte injury in KOA via the MAPK signaling pathway.
\end{abstract}

\section{Introduction}

Knee osteoarthritis (KOA) is a common degenerative joint disease caused by several factors, such as age, obesity, inflammation and trauma, and is characterized by the degeneration of articular cartilage (1). KOA results in the destruction of

Correspondence to: Dr Bojie Chen, Department of Joint Surgery, Ganzhou People's Hospital, 16 Meiguan Avenue, Zhanggong, Jiangxi 341000, P.R. China

E-mail: chenbojie20@163.com

Key words: knee osteoarthritis, dual specificity protein phosphatase 4, lipopolysaccharide-induced chondrocyte injury, MAPK signaling pathway articular cartilage, and triggers pathological changes in the surrounding synovial membrane, ligaments and soft tissue (2). These pathological changes result in the damage of articular cartilage and the potential destruction of the entire articular surface (3). At present, there are no available strategies for the prevention and treatment of KOA. Thus, further investigations into the mechanisms underlying KOA may provide novel treatment strategies, and research into the diagnosis of KOA and further treatment options is ongoing in a number of countries (4).

Dual specificity protein phosphatase 4 (DUSP4), also known as MAPK phosphatase 2 (MKP2), is a member of the DUSP family (5). DUSP4 serves an important role in the regulation of cell proliferation and differentiation (6). As a negative regulator of the MAPK signaling pathway, DUSP4 also plays a tumor-suppressive role in the majority of human cancer types, including colorectal, breast and pancreatic cancer (7). The results of a previous study demonstrated that DUSP4 overexpression in vascular endothelial cells reduced oxidative stress-induced cell damage and apoptosis to protect the heart following cardiac ischemia or reperfusion (8). In lipopolysaccharide (LPS)-induced acute lung injury, interference with DUSP4 expression promoted the expression of inflammatory cytokines, such as TNF $\alpha$, IL-1 and IL-6 (9). These results suggested that DUSP4 may serve an important role in the regulation of cell inflammation, injury and apoptosis in numerous diseases, including cancer, and inflammatory and vascular diseases. Osteoarthritis (OA) is characterized by the progressive degeneration and loss of articular cartilage caused by chondrocyte apoptosis and excessive degradation of the cartilage matrix. Cellular inflammatory factors are the main factors that promote excessive degradation of the cartilage matrix (10). Furthermore, the results of a previous study demonstrated that antisense long-non coding RNA in the INK4 locus regulated the proliferation and apoptosis of OA-related cells via the microRNA-122-5p/DUSP4 axis, indirectly suggesting that DUSP4 may be involved in the occurrence of OA (11). However, the specific mechanism underlying DUSP4 in $\mathrm{KOA}$ is yet to be fully elucidated.

In the present study, chondrocytes were induced with LPS to establish an OA injury model. Furthermore, the expression of DUSP4 was determined in the OA injury model to uncover the regulatory mechanism underlying DUSP4, thus providing novel insights into the occurrence and development of KOA. 


\section{Materials and methods}

Cell culture and model induction. Murine cartilage ATDC5 cells were purchased from the Institute of Biochemistry and Cell Biology at the Chinese Academy of Sciences. Cells were maintained in DMEM (Gibco; Thermo Fisher Scientific, Inc.) supplemented with 10\% FBS (Gibco; Thermo Fisher Scientific, Inc.) at $37^{\circ} \mathrm{C}$ in a humidified incubator containing $5 \% \mathrm{CO}_{2}$. Cells were pretreated with $100 \mathrm{ng} / \mathrm{ml}$ phorbol 12-myristate 13-acetate (PMA; cat. no. HY-18739; MedChemExpress) for $2 \mathrm{~h}$ and then treated with $5 \mu \mathrm{g} / \mathrm{ml} \mathrm{LPS}$ for $24 \mathrm{~h}$ at $37^{\circ} \mathrm{C}$, while untreated cells served as the control group.

GSE1919 microarray analysis. The Gene Expression Omnibus (GEO) database (https://www.ncbi-nlm-nih-gov. elib.tcd.ie/geo/) was used to search for the key word 'Osteoarthritis', and the GSE1919 chip data were selected for further analysis (12). The healthy samples $(n=5)$ and OA samples $(n=5)$ were selected. The data were analyzed using GEO2R online tools, and GraphPad Prism version 8 (GraphPad Software, Inc.) was used to aggregate the data into a histogram.

Reverse transcription-quantitative (RT- $q) P C R$. Total RNA was extracted from ATDC5 cells using TRIzol ${ }^{\circledR}$ reagent (Invitrogen; Thermo Fisher Scientific, Inc.), according to the manufacturer's protocols. Total RNA was reverse transcribed into cDNA using the PrimeScript RT Reagent kit (Takara Bio, Inc.). cDNA was synthesized from total RNA $(0.5 \mu \mathrm{g})$ at $48^{\circ} \mathrm{C}$ for $30 \mathrm{~min}$, followed by $95^{\circ} \mathrm{C}$ for $10 \mathrm{~min}$. The relative Mrna expression levels were determined using the One Step SYBR ${ }^{\circledR}$ PrimeScript ${ }^{\circledR}$ PLUS RT-RNA PCR Kit (Takara Biotechnology Co., Ltd.). The synthesized cDNA $(0.7 \mu \mathrm{g})$ was subjected to qPCR under the following thermocycling conditions: Initial denaturation at $95^{\circ} \mathrm{C}$ for $15 \mathrm{~min}$, followed by $45 \mathrm{cycles}$ of $95^{\circ} \mathrm{C}$ for $10 \mathrm{sec}, 66^{\circ} \mathrm{C}$ for $20 \mathrm{sec}$, and $72^{\circ} \mathrm{C}$ for $10 \mathrm{sec}$, according to the manufacturer's instructions. GAPDH was used to normalize the expression levels of the target genes and data were calculated by the $2^{-\Delta \Delta \mathrm{Cq}}$ method (13). The primer sequences used for qPCR were as follows: DUSP4 forward, 5'-TGGTTCATGGAAGCCATAGAG-3' and reverse, 5'-CCC GTTTCTTCATCATCAGG-3'; and GAPDH forward, 5'-AAC TTTGGCATTGTGGAAGG-3' and reverse, 5'-GGAGAC AACCTGGTCCTCAG-3'.

Western blot analysis. Total proteins were extracted from ATDC5 cells using RIPA buffer (Beijing Solarbio Science $\&$ Technology Co., Ltd.) and the protein concentration was determined using a BCA protein assay kit (Beijing Solarbio Science \& Technology Co., Ltd.). Subsequently, $30 \mu \mathrm{g} / \mathrm{lane}$ of total protein extracts were separated via SDS-PAGE on $10 \%$ gel and transferred onto nitrocellulose membranes. Following blocking with 5\% non-fat milk for $1 \mathrm{~h}$ at room temperature, the membranes were incubated with the following primary antibodies: anti-DUSP4 (1:1,000; cat. no. ab216576; Abcam), anti-p38 (1:1,000; cat. no. ab170099; Abcam), anti-phosphorylated (p)-p38 (1:1,000; cat. no. ab178867; Abcam), anti-ERK1/2 (1:1,000; cat. no. ab184699; Abcam), anti-p-ERK1/2 (1:1,000, cat. no. ab278538; Abcam), anti-JNK (1:1,000; cat. no. ab110724; Abcam),
anti-p-JNK (1:1,000; cat. no. ab215208; Abcam), anti-IL-6 $(1: 1,000$; cat. no. ab233706; Abcam), anti-TNF- $\alpha$ (1:1,000; cat. no. ab183218; Abcam), anti-IL-1 $\beta$ (1:1,000; cat. no. ab216995; Abcam), anti-Bcl-2 (1:1,000; cat. no. ab32124; Abcam), anti-Bax (1:1,000; cat. no. ab182733; Abcam), anti-cleaved caspase 3 (1:1,000; cat. no. ab32042; Abcam), anti-caspase 3 (1:1,000; cat. no. ab32351; Abcam) and GAPDH $\left(1: 1,000\right.$; cat. no. ab8245; Abcam) at $4^{\circ} \mathrm{C}$ overnight. Following washing with PBS containing $0.05 \%$ Tween-20 (1,000 ml PBS and $5 \mathrm{ml}$ Tween-20) three times, the membranes were incubated with the corresponding HRP-conjugated goat anti-rabbit (1:5,000; cat. no. LK2001; Sungene Biotech Co., Ltd.) or anti-mouse $(1: 5,000$, cat. no. LK2003; Sungene Biotech Co., Ltd.) secondary antibodies for $1 \mathrm{~h}$ at room temperature. The immunoblots were detected with an ECL kit (Advansta, Inc.) and the intensity of the bands was analyzed using ImageJ software (version 146; National Institutes of Health). GAPDH served as the loading control for normalization.

Cell transfection. The DUSP4 overexpression plasmid (ov-DUSP4) was designed and synthesized by Shanghai GeneChem Co., Ltd., using the pBluescript vector. A total of $1 \mu \mathrm{g}$ ov-DUSP4 plasmid or an empty vector (NC) were transfected into ATDC5 cells using Lipofectamine ${ }^{\circledR} 2000$ at $37^{\circ} \mathrm{C}$ for $48 \mathrm{~h}$ (Invitrogen; Thermo Fisher Scientific, Inc.), according to the manufacturer's protocol. Following incubation for $48 \mathrm{~h}$, cells were used for subsequent experiments.

Cell Counting Kit-8 (CCK-8). ATDC5 cells were seeded into 96-well microplates at a density of $1 \times 10^{4}$ cells/well at $37^{\circ} \mathrm{C}$ for $12 \mathrm{~h}$. Following cell transfection and treatment with LPS and PMA, $10 \mu$ l CCK-8 reagent (Thermo Fisher Scientific, Inc.) was added to each well and cells were cultured at $37^{\circ} \mathrm{C}$ for $4 \mathrm{~h}$. The Infinite ${ }^{\mathrm{TM}}$ M200 microplate reader (Tecan Group, Ltd.) was utilized to measure the optical density of each well at a wavelength of $460 \mathrm{~nm}$.

Reactive oxygen species (ROS), lactate dehydrogenase $(L D H)$, superoxide dismutase (SOD) and malondialdehyde (MDA) assays. The levels of ROS, LDH, SOD and MDA were determined using the ROS/Superoxide Detection Assay kit (cat. no. ab113851; Abcam), LDH cytotoxicity assay kit (cat. no. C0017, Beyotime Institute of Biotechnology), total SOD assay kit (cat. no. BC0175, Beijing Solarbio Science \& Technology Co., Ltd.) and MDA kit (cat. no. E0597Ge, Wuhan EIAab Science Co., Ltd.) respectively, according to the corresponding manufacturers' protocols.

ELISA. The levels of IL-1 $\beta$ (cat. no. MLB00C), IL-6 (cat. no. D6050) and TNF- $\alpha$ (cat. no. MTA00B) in the cell supernatant were measured using the IL- $1 \beta$, IL- 6 and TNF- $\alpha$ ELISA kits (R\&D Systems, Inc.), according to the manufacturer's protocol.

TUNEL assay. Cell apoptosis was evaluated using a TUNEL assay kit (Beyotime Institute of Biotechnology) according to the manufacturer's protocol. The cells were fixed with Immunol Staining Fix Solution (Beyotime Institute of Biotechnology) for $30 \mathrm{~min}$ at $37^{\circ} \mathrm{C}$, followed by washing 

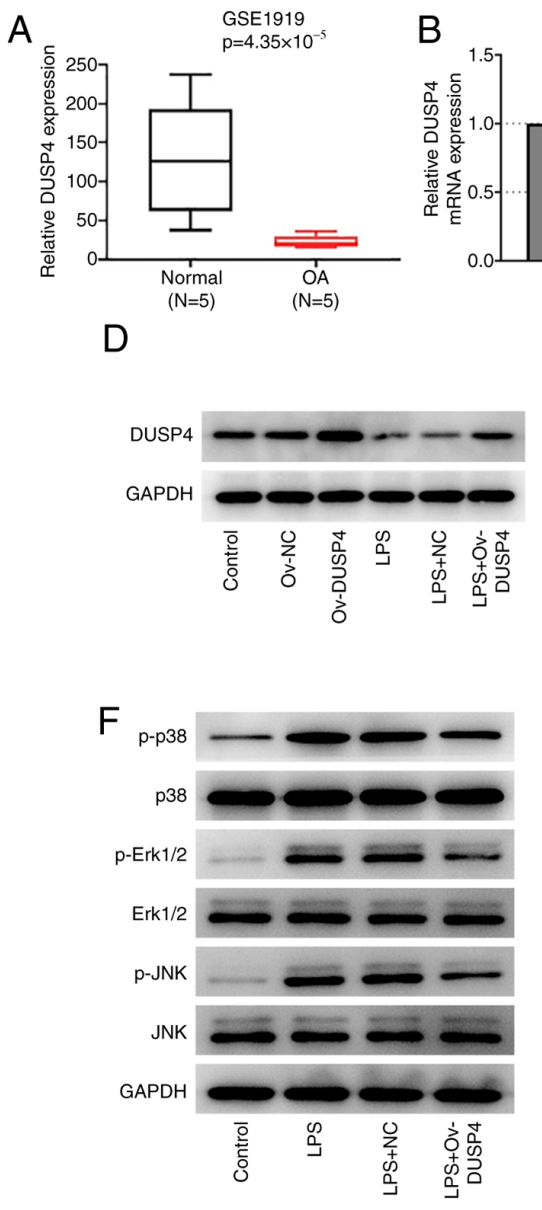
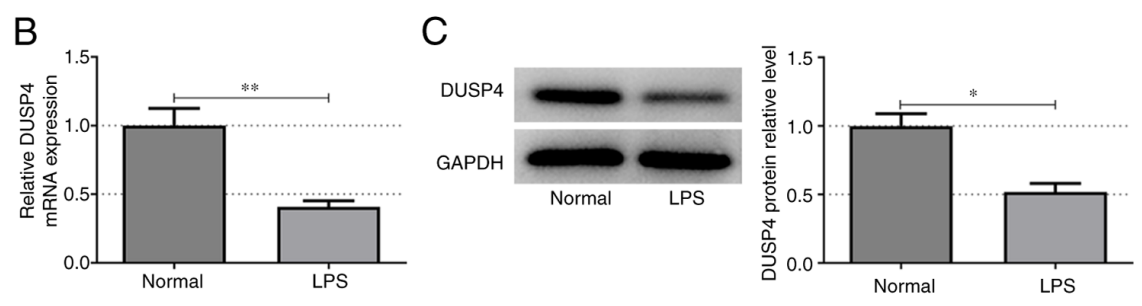

E
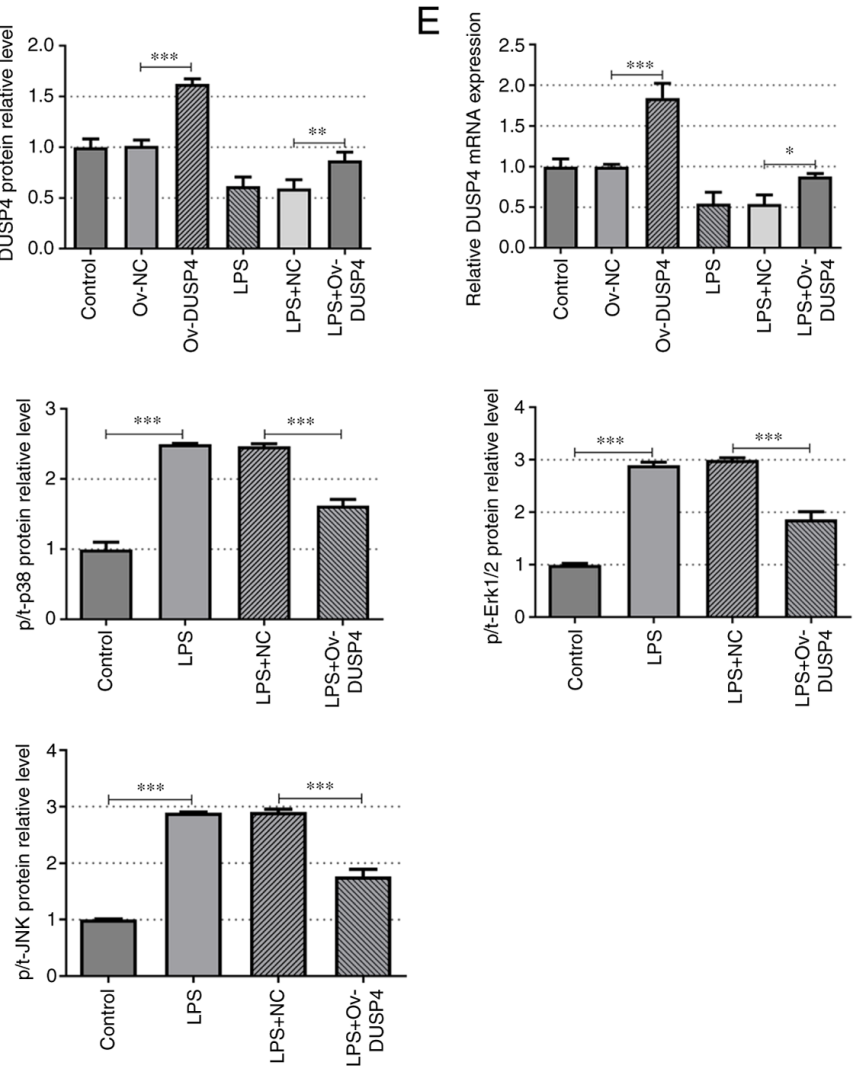

Figure 1. DUSP4 is expressed at low levels in OA chondrocytes, and upregulation of DUSP4 inhibits the MAPK signaling pathway. (A) The expression of DUSP4 in synovial tissues of patients with OA was revealed using GSE1919 chip data analysis. (B) RT-qPCR and (C) western blotting were used to detect the expression level of DUSP4. (D) Western blot analysis revealed the expression level of DUSP4 following cell transfection with Ov-DUSP4. (E) RT-qPCR was used to detect the expression level of DUSP4 following cell transfection. (F) Western blotting was used to detect the expression levels of MAPK signaling pathway proteins. ${ }^{*} \mathrm{P}<0.05 ;{ }^{* *} \mathrm{P}<0.01 ;{ }^{* * *} \mathrm{P}<0.001$. OA, osteoarthritis; DUSP4, dual specificity protein phosphatase 4; LPS, lipopolysaccharide; Ov, overexpression; NC, negative control; $\mathrm{p}$-, phosphorylated; t-, total; RT-qPCR, reverse transcription-quantitative PCR.

with PBS (Thermo Fisher Scientific, Inc.). Subsequently, cells were incubated with PBS containing $0.3 \%$ Triton X-100 (Sigma-Aldrich; Merck KGaA) at room temperature for 5 min and were washed again with PBS followed by TUNEL solution for $1 \mathrm{~h}$ at $37^{\circ} \mathrm{C}$. Apoptotic cell nuclei were stained with fluorescein-labeled dUTP solution (Invitrogen; Thermo Fisher Scientific, Inc.) at $37^{\circ} \mathrm{C}$ for $60 \mathrm{~min}$, while all cell nuclei were stained with $5 \mathrm{mg} / \mathrm{ml}$ DAPI for $3 \mathrm{~min}$ at $37^{\circ} \mathrm{C}$. A total of 10 fields were randomly selected and observed by fluorescence microscope (magnification, x200; CKX53; Olympus Corporation). TUNEL-positive staining indicated apoptotic cells.

Statistical analysis. All data are expressed as the mean \pm standard deviation. One-way ANOVA followed by Tukey's post hoc test was used for comparisons among multiple groups. $\mathrm{P}<0.05$ was considered to indicate a statistically significant difference. All analyses were carried out using SPSS 22.0 statistical software (IBM Corp.). Each experiment was repeated three times.

\section{Results}

DUSP4 is downregulated in OA chondrocytes and DUSP4 overexpression inhibits the MAPK signaling pathway. GSE1919 microarray analysis revealed that DUSP4 was significantly downregulated in synovial tissues of patients with OA (Fig. 1A). Additionally, in vitro experiments demonstrated that the expression of DUSP4 was significantly decreased in LPS-induced ATDC5 cells compared with the control group at both the protein and mRNA level (Fig. 1B and C). These results indicated that the expression of DUSP4 was decreased in OA chondrocytes. The ov-DUSP4 plasmid was constructed and the subsequent transfection efficiency was verified by RT-qPCR and western blot analyses in ATDC5 cells. DUSP4 was significantly overexpressed in ATDC5 cells and LPS-induced ATDC5 cells compared with the corresponding control group at both the protein and mRNA level (Fig. 1D and E). Subsequently, cells were divided into four groups: i) Control; ii) LPS; iii) LPS + NC; and iv) LPS + 

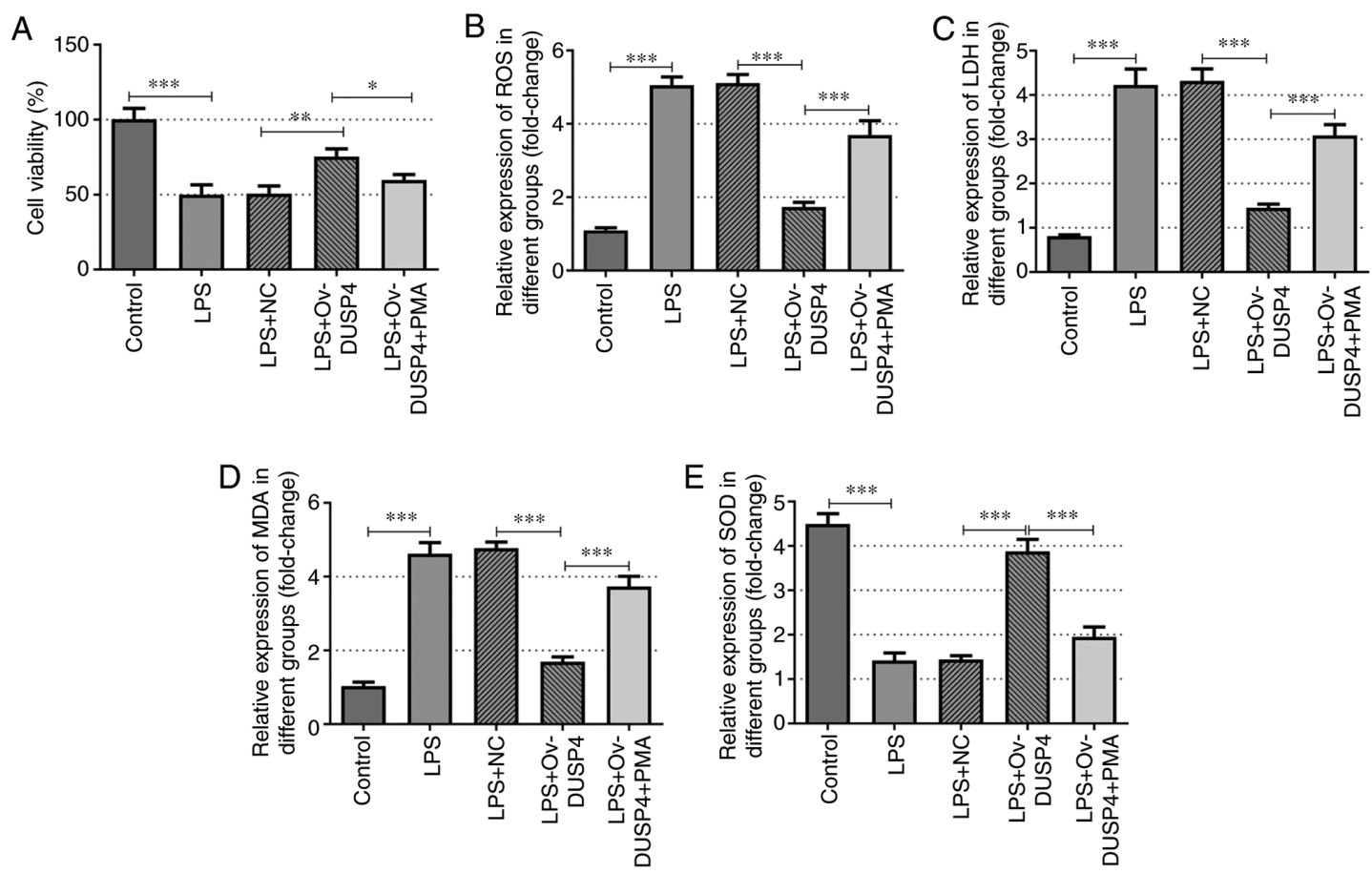

Figure 2. DUSP4 increases the activity and reduces the oxidative stress level of osteoarthritis chondrocytes via the MAPK signaling pathway. (A) MTT assays were used to detect the cell viability. ELISA assays detected the expression of (B) ROS, (C) LDH, (D) MDA and (E) SOD. ${ }^{*}<<0.05$; ${ }^{* *} \mathrm{P}<0.01 ;{ }^{* * *} \mathrm{P}<0.001$. LPS, lipopolysaccharide; NC, negative control; Ov, overexpression; DUSP4, dual specificity protein phosphatase 4; PMA, phorbol 12-myristate 13-acetate; ROS, reactive oxygen species; $\mathrm{LDH}$, lactate dehydrogenase; MDA, malondialdehyde; SOD, superoxide dismutase.

ov-DUSP4. The expression of MAPK pathway-related proteins was assessed by western blot analysis. The expression levels of p-p38, p-ERK1/2 and p-JNK were significantly increased in the LPS group compared with the control group. Furthermore, the expression of p-p38, p-ERK1/2 and p-JNK was significantly downregulated following DUSP4 overexpression, compared with the LPS + NC group (Fig. 1F). The results of the present study suggested that DUSP4 may serve an important role in promoting chondrocyte injury in OA by inhibition of the MAPK signaling pathway.

DUSP4 enhances cell viability and attenuates oxidative stress and inflammatory responses in OA chondrocytes via the MAPK signaling pathway. To further investigate whether DUSP4 exerts its regulatory effects on OA via the MAPK signaling pathway, cells were treated with PMA, an agonist of the MAPK signaling pathway. Cells were divided into five groups: i) Control; ii) LPS; iii) LPS + NC; iv) LPS + ov-DUSP4; and v) LPS + ov-DUSP4 + PMA. A CCK-8 assay was performed to assess cell viability and the results demonstrated that cell viability was significantly reduced in the LPS group compared with the control group. Furthermore, cell viability in the LPS + ov-DUSP4 group was significantly increased compared with the LPS + NC group, and was significantly attenuated following cell treatment with PMA compared with the LPS + ov-DUSP4 group (Fig. 2A).

The generation of ROS, the levels of LDH and MDA and the activity of SOD in cells were determined by ELISA. The results demonstrated that the levels of ROS, LDH and MDA was significantly enhanced in the LPS group, while the activity of SOD was markedly decreased compared with the control group. Additionally, the production of ROS, LDH and MDA was significantly diminished in the LPS + ov-DUSP4 group, whereas the production of SOD was significantly enhanced, compared with the LPS + NC group. In addition, the production of ROS, LDH and MDA was notably decreased in the LPS + ov-DUSP4 group compared with the LPS + ov-DUSP4 + PMA group, while the opposite trend was observed in the activity of SOD (Fig. 2B-E). Furthermore, the secretion levels of the inflammation-related factors, IL-6, TNF- $\alpha$ and IL- $1 \beta$ were measured in each group using ELISA. The results demonstrated that PMA reversed the inhibitory effect of DUSP4 overexpression on the protein expression levels of IL-6, TNF- $\alpha$ and IL-1 $\beta$ compared with the LPS + ov-DUSP4 group (Fig. 3A and B).

DUSP4 alleviates OA chondrocyte apoptosis via the MAPK signaling pathway. TUNEL and western blotting assays were carried out to evaluate cell apoptosis. Compared with the control group, cell apoptosis was significantly increased in the LPS group, accompanied by upregulation of Bax and cleaved caspase 3 , and the significant downregulation of Bcl-2. Additionally, apoptosis was reduced in the LPS + ov-DUSP4 group compared with the LPS + NC group. Consistent with the aforementioned results, the expression of Bax and cleaved caspase 3 was decreased, while that of $\mathrm{Bcl}-2$ was increased in the LPS + ov-DUSP4 group compared with the LPS + NC group. The number of apoptotic cells and the expression of Bax and cleaved caspase 3 were increased in the LPS + ov-DUSP4 + PMA group compared with the LPS + ov-DUSP4 group. However, Bcl-2 was downregulated in the LPS + ov-DUSP4 + PMA group compared with the LPS + ov-DUSP4 group (Fig. 4A and B). These results indicated that DUSP4 alleviated apoptosis of OA chondrocyte by inhibition of the MAPK signaling pathway. 
A
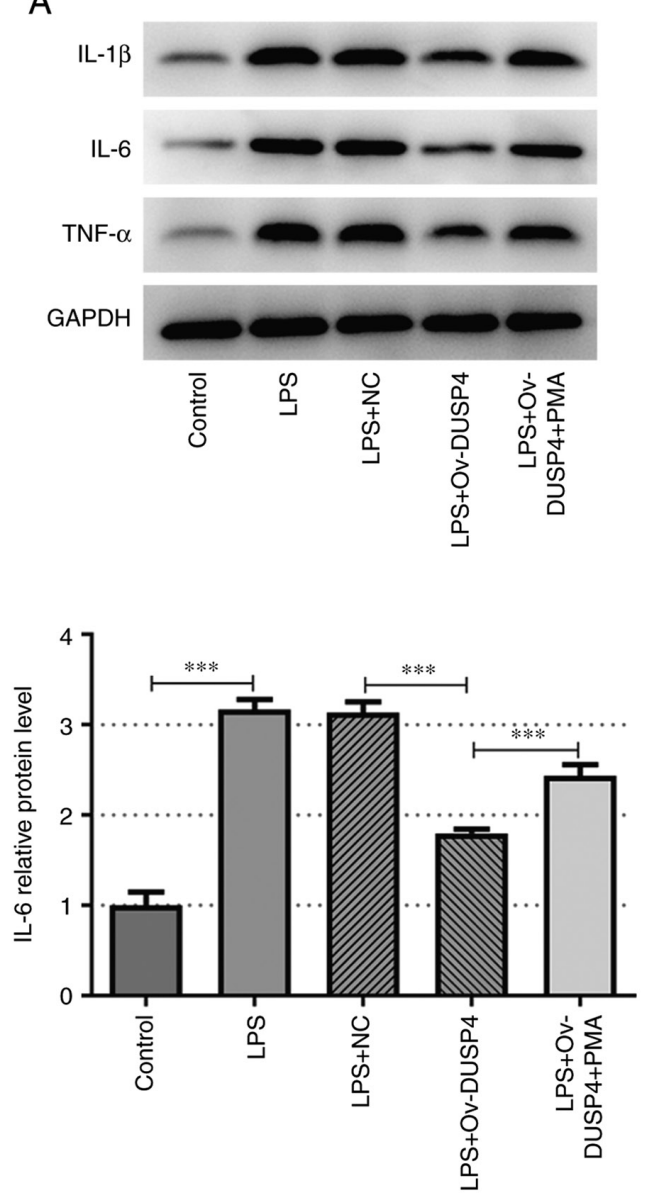
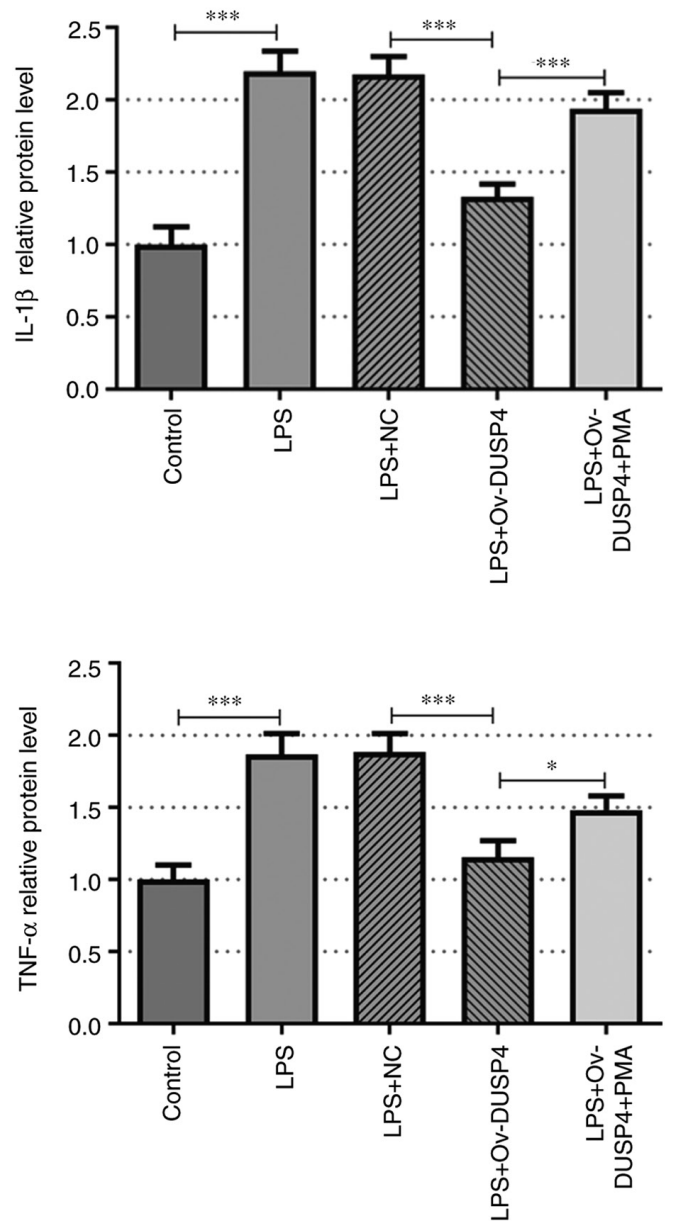

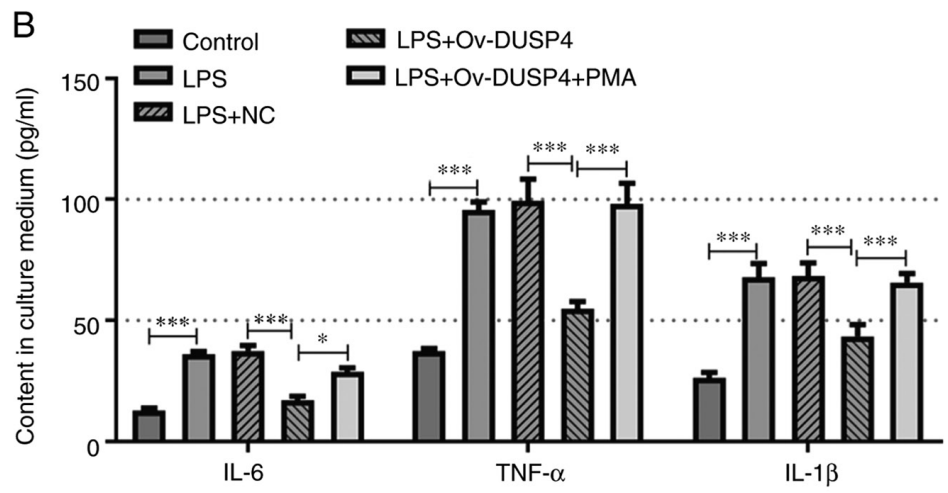

Figure 3. DUSP4 reduces the inflammatory response of osteoarthritis chondrocytes via the MAPK signaling pathway. (A) Western blot analysis was used to detect the expression levels of inflammatory-related proteins, IL-6, TNF- $\alpha$ and IL-1 $\beta$. (B) ELISA assays were used to detect the expression levels of IL-6, TNF- $\alpha$ and IL-1 $\beta .{ }^{*} \mathrm{P}<0.05 ;{ }^{* * *} \mathrm{P}<0.001$. LPS, lipopolysaccharide; NC, negative control; Ov, overexpression; DUSP4, dual specificity protein phosphatase 4; PMA, phorbol 12-myristate 13-acetate.

\section{Discussion}

OA involves a number of complex mechanisms, including cartilage degradation, synovitis, and destruction and loss of articular cartilage (14). Chondrocytes, the only specialized cell type found in cartilage tissue, serve a critical role in maintaining joint stability (15). As prochondrocytes, ATDC5 cells differentiate in a similar way to cells that undergo chondrogenesis (16). ATDC5 cells are used as in vitro phytopharmacological models to reflect the complete process of chondrocyte proliferation, differentiation and mineralization during chondrogenesis (17). At present, the application of ATDC5 cells in OA research has been widely accepted (18-20). In the present study, ATDC5 cells were induced with LPS to establish an OA cell model, as previously described $(20,21)$. In the present study, the mouse OA chondrocyte injury model was utilized to explore the regulatory role and mechanism underlying DUSP4 in chondrocyte injury. However, the limitations of the present study include limited cell line selection, leading to a lack of analysis in human chondrocytes. Human chondrocytes will be used for verification in future experiments. 
A
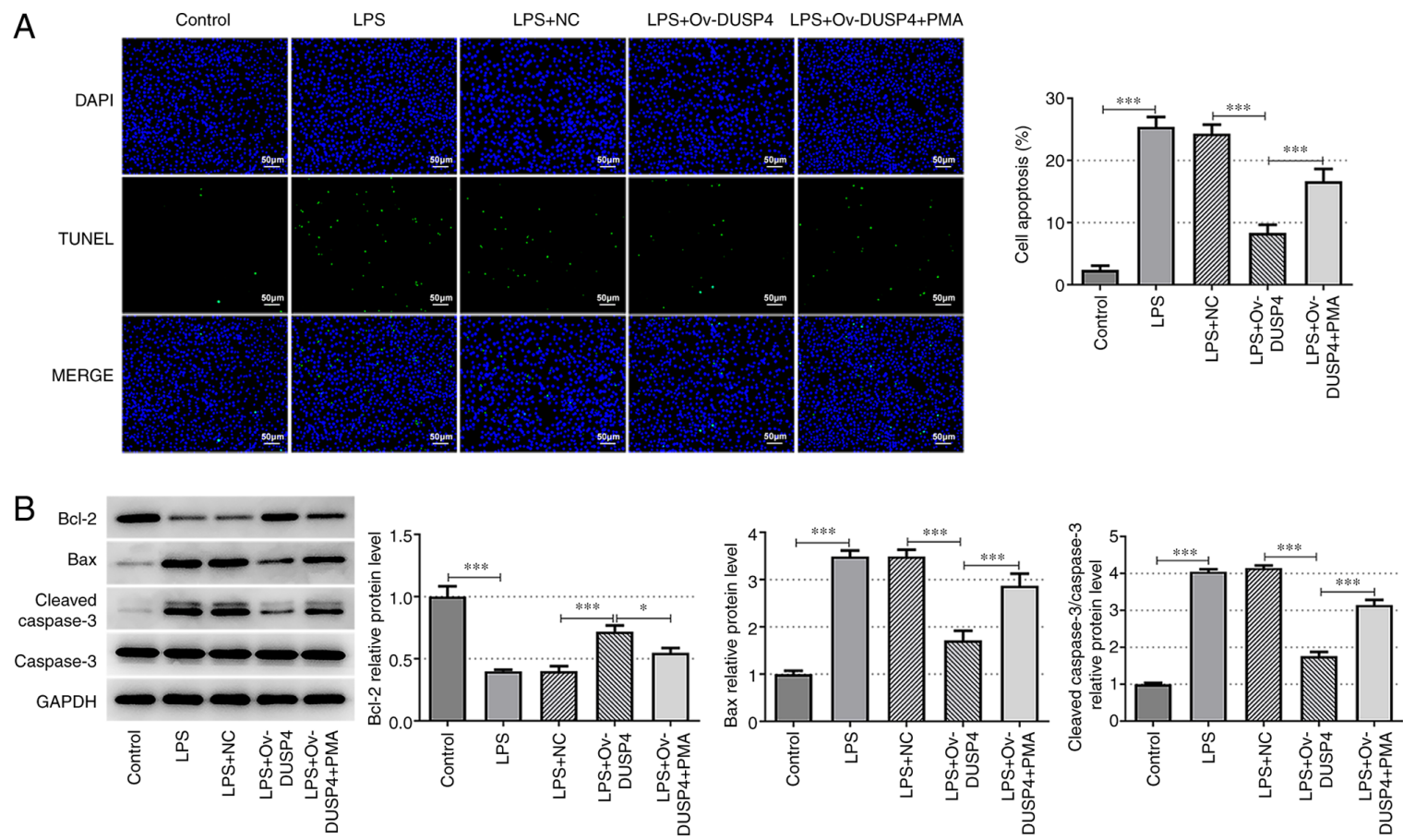

Figure 4. DUSP4 alleviates apoptosis of osteoarthritis chondrocytes via the MAPK signaling pathway. (A) TUNEL assay was performed to detect the levels of apoptosis. (B) Western blot analysis was used to detect the expression levels of Bcl-2, Bax, caspase 3 and cleaved caspase 3. ${ }^{*} \mathrm{P}<0.05$; ${ }^{* * * *} \mathrm{P}<0.001$. LPS, lipopolysaccharide; NC, negative control; Ov, overexpression; DUSP4, dual specificity protein phosphatase 4; PMA, phorbol 12-myristate 13-acetate.

Emerging evidence has demonstrated that DUSP4 serves a regulatory role in cell inflammation, injury and apoptosis in a variety of diseases $(9,22,23)$. However, the exact mechanism underlying DUSP4 in OA is yet to be elucidated. In the present study, results of the GSE1919 dataset analysis demonstrated that the expression of DUSP4 was notably downregulated in OA. In addition, the expression of DUSP4 was also significantly decreased in LPS-induced ATDC5 cells. DUSP4, also known as MKP2, serves a key role as a negative regulator of the MAPK signaling pathway in a number of biological processes (24). Rapid phosphorylation of ERK1/2 following LPS induction in microglia induces the expression of DUSP1, DUSP4, DUSP2 and DUSP5 (25). The result demonstrated that DUSPs exhibit different substrate preferences for specific MAPKs. In the present study, phosphorylation of $\mathrm{p} 38$, ERK1/2 and JNK in the MAPK signaling pathway resulted in decreased expression of DUSP4 following LPS induction. These results indicated that LPS inhibited the expression of DUSP4 by promoting p38, ERK1/2 and JNK phosphorylation. Li et al (11) demonstrated that regulation of DUSP4 expression enhanced the proliferation and apoptosis of OA cells, thus suggesting that DUSP4 may be involved in the occurrence of OA. In the present study, DUSP4 overexpression markedly enhanced LPS-induced ATDC5 cell viability and attenuated oxidative stress, cell apoptosis and inflammatory response.

DUSP4 dephosphorylates several key signaling molecules, including those involved in the MAPK signaling pathway (26). The results of a previous study indicated that DUSP4 silencing in diabetes enhanced the activity of the p38/JNK signaling pathway (27). Additionally, simultaneously activating the MAPK signaling pathway aggravated LPS-induced chondrocyte injury (28). Ham et al (25) revealed an association between LPS, DUSPs and ERK1/2 in microglia cells, and highlighted that LPS induced ERK1/2 phosphorylation, thereby regulating the expression of DUSP4 and 6. Inhibition of ERK1/2 phosphorylation also inhibited LPS-induced DUSP4 expression. In addition, inhibition of DUSP6 increased ERK1/2 phosphorylation (25). However, the role of DUSP4 in ERK1/2 phosphorylation and regulation of the MAPK signaling pathway are yet to be fully elucidated. Thus, we hypothesized that DUSP4 attenuated LPS-induced chondrocyte injury in the KOA model via inhibition of the MAPK signaling pathway. To further verify this hypothesis, cells were co-treated with the MAPK signaling pathway agonist, PMA. The results demonstrated that PMA reversed the inhibitory effect of DUSP4 overexpression on oxidative stress, inflammatory response and LPS-induced ATDC5 cell apoptosis. The aforementioned findings suggested that DUSP4 alleviated LPS-induced chondrocyte injury in the KOA model via inhibition of the MAPK signaling pathway.

Further limitations of the present study include the investigation of DUSP4 expression at a cellular level, as the mechanisms underlying the effects of DUSP4 on OA were not evaluated in vivo. Therefore, the effects of DUSP4 on OA must be further verified using in vivo experiments. In addition, it is unclear whether DUSP4 plays a role in OA by targeting the MAPK signaling pathway, or by targeting alternate genes that regulate the MAPK pathway.

The detection of inflammatory-related factors and apoptosis-related indicators were the main focus of the present study. In following experiments, the roles of these factors will be further investigated.

In conclusion, the present study demonstrated that DUSP4 alleviated LPS-induced chondrocyte injury in KOA via inhibition of the MAPK signaling pathway. The present study provided a theoretical basis for further investigation into the mechanisms underlying the development of KOA. 


\section{Acknowledgements}

The authors would like to acknowledge Ganzhou Key Laboratory of Bone and Joint (Jiangxi, China), which offered help in the procurement of experimental materials and data collection, and was used to perform experiments in the present study.

\section{Funding}

The present study was supported by the Science and Technology Plan of Jiangxi Provincial Health Commission (grant no. 20204611).

\section{Availability of data and materials}

The datasets used and/or analyzed during the current study are available from the corresponding author on reasonable request.

\section{Authors' contributions}

ZL wrote the manuscript, analyzed the data, performed the experiments and supervised the study. BC searched the literature, revised the manuscript for important intellectual content, made substantial contributions to the conception and design of the study, and contributed to analysis and interpretation of data. ZL and BC confirm the authenticity of all the raw data. All authors have read and approved the final manuscript.

\section{Ethics approval and consent to participate}

Not applicable.

\section{Patient consent for publication}

Not applicable.

\section{Competing interests}

The authors declare that they have no competing interests.

\section{References}

1. Kan HS, Chan PK, Chiu KY, Yan CH, Yeung SS, Ng YL, Shiu KW and Ho T: Non-surgical treatment of knee osteoarthritis. Hong Kong Med J 25: 127-133, 2019.

2. Mathiessen A and Conaghan PG: Synovitis in osteoarthritis: Current understanding with therapeutic implications. Arthritis Res Ther 19: 18, 2017.

3. Jeffries MA: Osteoarthritis year in review 2018: Genetics and epigenetics. Osteoarthritis Cartilage 27: 371-377, 2019.

4. Barnett R: Osteoarthritis. Lancet 391: 1985, 2018.

5. Low HB and Zhang Y: Regulatory roles of MAPK phosphatases in cancer. Immune Netw 16: 85-98, 2016

6. Ratsada P, Hijiya N, Hidano S, Tsukamoto Y, Nakada C, Uchida T, Kobayashi $\mathrm{T}$ and Moriyama M: DUSP4 is involved in the enhanced proliferation and survival of DUSP4-overexpressing cancer cells. Biochem Biophys Res Commun 528: 586-593, 2020.

7. Kang X, Li M, Zhu H, Lu X, Miao J, Du S, Xia X and Guan W: DUSP4 promotes doxorubicin resistance in gastric cancer through epithelial-mesenchymal transition. Oncotarget 8 94028-94039, 2017

8. Barajas-Espinosa A, Basye A, Angelos MG and Chen CA: Modulation of p38 kinase by DUSP4 is important in regulating cardiovascular function under oxidative stress. Free Radic Biol Med 89: 170-181, 2015
9. Lu Z, Feng H, Shen X, He R, Meng H, Lin W and Geng Q: MiR-122-5p protects against acute lung injury via regulation of DUSP4/ERK signaling in pulmonary microvascular endothelial cells. Life Sci 256: 117851, 2020.

10. Varela-Eirin M, Loureiro J, Fonseca E, Corrochano S, Caeiro JR, Collado $\mathrm{M}$ and Mayan MD: Cartilage regeneration and ageing: Targeting cellular plasticity in osteoarthritis. Ageing Res Rev 42: 56-71, 2018.

11. Li X, Huang TL, Zhang GD, Jiang JT and Guo PY: LncRNA ANRIL impacts the progress of osteoarthritis via regulating proliferation and apoptosis of osteoarthritis synoviocytes. Eur Rev Med Pharmacol Sci 23: 9729-9737, 2019.

12. Giovannini-Chami L, Marcet B, Moreilhon C, Chevalier B, Illie MI, Lebrigand K, Robbe-Sermesant K, Bourrier T, Michiels JF, Mari B, et al: Distinct epithelial gene expression phenotypes in childhood respiratory allergy. Eur Respir J 39: 1197-1205, 2012.

13. Livak KJ and Schmittgen TD: Analysis of relative gene expression data using real-time quantitative PCR and the 2(-Delta Delta C(T)) method. Methods 25: 402-408, 2001.

14. Martel-Pelletier J, Barr AJ, Cicuttini FM, Conaghan PG, Cooper C, Goldring MB, Goldring SR, Jones G, Teichtahl AJ and Pelletier JP: Osteoarthritis. Nat Rev Dis Primers 2: 16072, 2016.

15. Charlier E, Deroyer C, Ciregia F, Malaise O, Neuville S, Plener Z, Malaise M and de Seny D: Chondrocyte dedifferentiation and osteoarthritis (OA). Biochem Pharmacol 165: 49-65, 2019.

16. Ogawa Y, Takahashi N, Takemoto T, Nishiume T, Suzuki M, Ishiguro N and Kojima T: Hyaluronan promotes TRPV4-induced chondrogenesis in ATDC5 cells. PLoS One 14: e0219492, 2019.

17. Wilhelm D, Kempf H, Bianchi A and Vincourt JB: ATDC5 cells as a model of cartilage extracellular matrix neosynthesis, maturation and assembly. J Proteomics 219: 103718, 2020.

18. Zhang H, Lin C, Zeng C, Wang Z, Wang H, Lu J, Liu X, Shao Y, Zhao C, Pan J, et al: Synovial macrophage M1 polarisation exacerbates experimental osteoarthritis partially through R-spondin-2. Ann Rheum Dis 77: 1524-1534, 2018.

19. Fan L, Li M, Cao FY, Zeng ZW, Li XB, Ma C, Ru JT and Wu XJ: Astragalus polysaccharide ameliorates lipopolysaccharide-induced cell injury in ATDC5 cells via miR-92a/KLF4 mediation. Biomed Pharmacother 118: 109180, 2019.

20. Liu M, Zhang J, Liu W and Wang W: Salidroside protects ATDC5 cells against lipopolysaccharide-induced injury through up-regulation of microRNA-145 in osteoarthritis. Int Immunopharmacol 67: 441-448, 2019.

21. Shu Y, Long J, Guo W and Ye W: MicroRNA-195-5p inhibitor prevents the development of osteoarthritis by targeting REG $\gamma$. Mol Med Rep 19: 4561-4568, 2019.

22. Westphal S, Stoppe C, Gruenewald M, Bein B, Renner J, Cremer J, Coburn M, Schaelte G, Boening A, Niemann B, et al: Genome-wide association study of myocardial infarction, atrial fibrillation, acute stroke, acute kidney injury and delirium after cardiac surgery-a sub-analysis of the RIPHeart-Study. BMC Cardiovasc Disord 19: 26, 2019.

23. Dougherty JA, Kilbane Myers J, Khan M, Angelos MG and Chen CA: Dual-specificity phosphatase 4 overexpression in cells prevents hypoxia/reoxygenation-induced apoptosis via the upregulation of eNOS. Front Cardiovasc Med 4: 22, 2017.

24. Kirchner A, Bagla S, Dachet F and Loeb JA: DUSP4 appears to be a highly localized endogenous inhibitor of epileptic signaling in human neocortex. Neurobiol Dis 145: 105073, 2020.

25. Ham JE, Oh EK, Kim DH and Choi SH: Differential expression profiles and roles of inducible DUSPs and ERK1/2-specific constitutive DUSP6 and DUSP7 in microglia. Biochem Biophys Res Commun 467: 254-260, 2015.

26. Chen HF, Chuang HC and Tan TH: Regulation of dual-specificity phosphatase (DUSP) ubiquitination and protein stability. Int J Mol Sci 20: 2668, 2019.

27. Denhez B, Rousseau M, Dancosst DA, Lizotte F, Guay A, Auger-Messier M, Côté AM and Geraldes P: Diabetes-induced DUSP4 reduction promotes podocyte dysfunction and progression of diabetic nephropathy. Diabetes 68: 1026-1039, 2019.

28. Lei J, Fu Y, Zhuang Y and Zhang K: Sema4D aggravated LPS-induced injury via activation of the MAPK signaling pathway in ATDC5 chondrocytes. Biomed Res Int 2020: 8691534, 2020.

This work is licensed under a Creative Commons Attribution-NonCommercial-NoDerivatives 4.0 International (CC BY-NC-ND 4.0) License. 